\title{
Intracranial Demyelinating Pseudotumor: A Case Report and Review of the Literature
}

\author{
Xianbin NING ${ }^{1,2}$, Changfu ZHAO², Caiqin WANG ${ }^{3}$, Duo ZHANG ${ }^{4}$, Qi LUO 1 \\ ${ }^{1}$ First Hospital of Jilin University, Department of Neurosurgery, Changchun, 130021, China \\ ${ }^{2}$ The Affiliated Hospital of Beihua University, Department of Neurosurgery, Jilin, 132011, China \\ ${ }^{3}$ The Affiliated Hospital of Beihua University, Department of Pathology, Jilin, 132011, China \\ ${ }^{4}$ The Affiliated Hospital of Beihua University, Department of Radiology, Jilin, 132011, China
}

Xianbin Ning and Changfu Zhao contributed equally to the work.

\section{ABSTRACT}

Demyelinating pseudotumor is a rare inflammatory demyelinating disease of the central nervous system (CNS) that has a similar clinical presentation and computed tomography (CT) and magnetic resonance imaging (MRI) imaging findings as brain tumors or abscesses. Unlike brain tumors, demyelinating pseudotumors respond well to steroid hormones. There are only a few reported cases of intracranial demyelinating pseudotumors in the literature. In this case report, we present the diagnosis and treatment of demyelinating pseudotumor in a patient whose condition was initially misdiagnosed as an astrocytoma. Based on the literature and our case, we formulated an outline for the differential diagnosis of demyelinating pseudotumor and astrocytoma. A timely and correct diagnosis of demyelinating pseudotumor would avoid blind surgery, radiotherapy and chemotherapy, which are used to treat brain tumors.

KEYWORDS: Demyelinating pseudotumor, Diagnosis, Cord-Like enhancement, Enhanced MRI

\section{INTRODUCTION}

$\mathrm{D}$ emyelinating pseudotumor of the brain, also called tumor-like demyelinating lesions (TDLs) or inflammatory demyelinating pseudotumor (IDP), is a rare inflammatory demyelinating disease of the central nervous system (CNS) $(3,4,7,8)$. The exact etiology of demyelinating pseudotumor is unclear, however it may associate with multiple sclerosis (MS) (5). Based on the clinical presentation and computed tomography (CT) and magnetic resonance imaging (MRI) findings, it is difficult to distinguish between brain tumors, abscesses and demyelinating pseudotumors (13). Unlike brain tumors, demyelinating pseudotumors respond well to steroid hormones (6). Therefore, a reliable distinction between brain tumors and intracranial demyelinating pseudotumors is important for a targeted therapy. Although the incidence of demyelinating pseudotumor in the clinic is relative low, delayed diagnosis or misdiagnosis may cause delays in treatment of these patients and subsequently may lead to physical and mental damage (14). The differential diagnosis of demyelinating pseudotumor and brain tumors, especially of low-grade astrocytomas, should arouse more attention to exclude the possibility of a demyelinating process. Despite this, only few reported cases of intracranial demyelinating pseudotumor are known in the literature. In this case report, we present the diagnosis and treatment of a demyelinating pseudotumor patient who was misdiagnosed with astrocytoma.

\section{CASE REPORT}

A female 40-year-old patient was hospitalized due to a progressive disability of the left limb that severely increased over one week. The patient did not have a recent history 
of fever or drug applications. The admission examination revealed that the patient was conscious, fluent, had a soft neck, light reflex sensitivity and hyperreflexia of the left knee, but the right side was normal. Her body temperature was $36.9^{\circ} \mathrm{C}$. Her pupils were the same size bilaterally and the pupillary light reflex was unaffected. The strength of the left and right limb muscle were graded as 3 and 5, respectively. A head CT showed a large low density area at the right parietal lobe and compressive deformation of the right lateral ventricle. A brain MRI with T1WI (T1 weighted image), T2WI and fluid attenuated inversion recovery (FLAIR) showed an abnormal signal at the right parietal lobe. Enhanced scans showed that the edge of the "lump" was in the sagittal plane and that a cord-like enhancement was distributed vertical to the lateral ventricles in the coronal plane (Figure 1A-D). A preoperative diagnosis of glioma was made, and craniotomy tumor resection was performed.

Intraoperative findings revealed that the "mass" had a yellowish foam texture, was avascular, and had clear boundaries to the surrounding tissue. The "mass" was completely resected and two weeks following the surgery, pathology showed that the "mass" was a demyelinating pseudotumor. Immunohisto- chemistry (IHC) revealed the following markers: Cluster of Difference 68 (CD68) (+), Myelin basic protein (MBP) (+), Vimentin (Vim) (+), Glial Fibrillary Acidic Protein (GFAP) (-) (Figure 3A-C). While waiting for the pathology report, the patient did not receive steroid hormone therapy. Three weeks after surgery, the hemiplegia became worse and the patient received steroids. The patient was treated with methylprednisolone pulse therapy $(1000 \mathrm{mg} / \mathrm{d})$, followed by oral prednisone $(1 \mathrm{mg} / \mathrm{kg})$ for two weeks. The patient was discharged from the hospital after two weeks. At the last examination, the left limb muscle strength was grade 4 , the right limb muscle strength was grade 5 , and the left knee showed active tendon reflexes and a left Babinski sign. Prednisone was given in tapering doses over a 2-month period. Follow-up was performed at 11 months postoperative, and the imaging is shown in Figure $2 A-D$. The patient recovered well, the left limb muscle strength was grade 4+ and the right limb muscle strength was grade 5 . The left knee tendon reflexes were normal and without a Babinski sign.

\section{DISCUSSION}

Demyelinating pseudotumor of the brain is a very rare demyelinating disease affecting the CNS. Previous reviews have

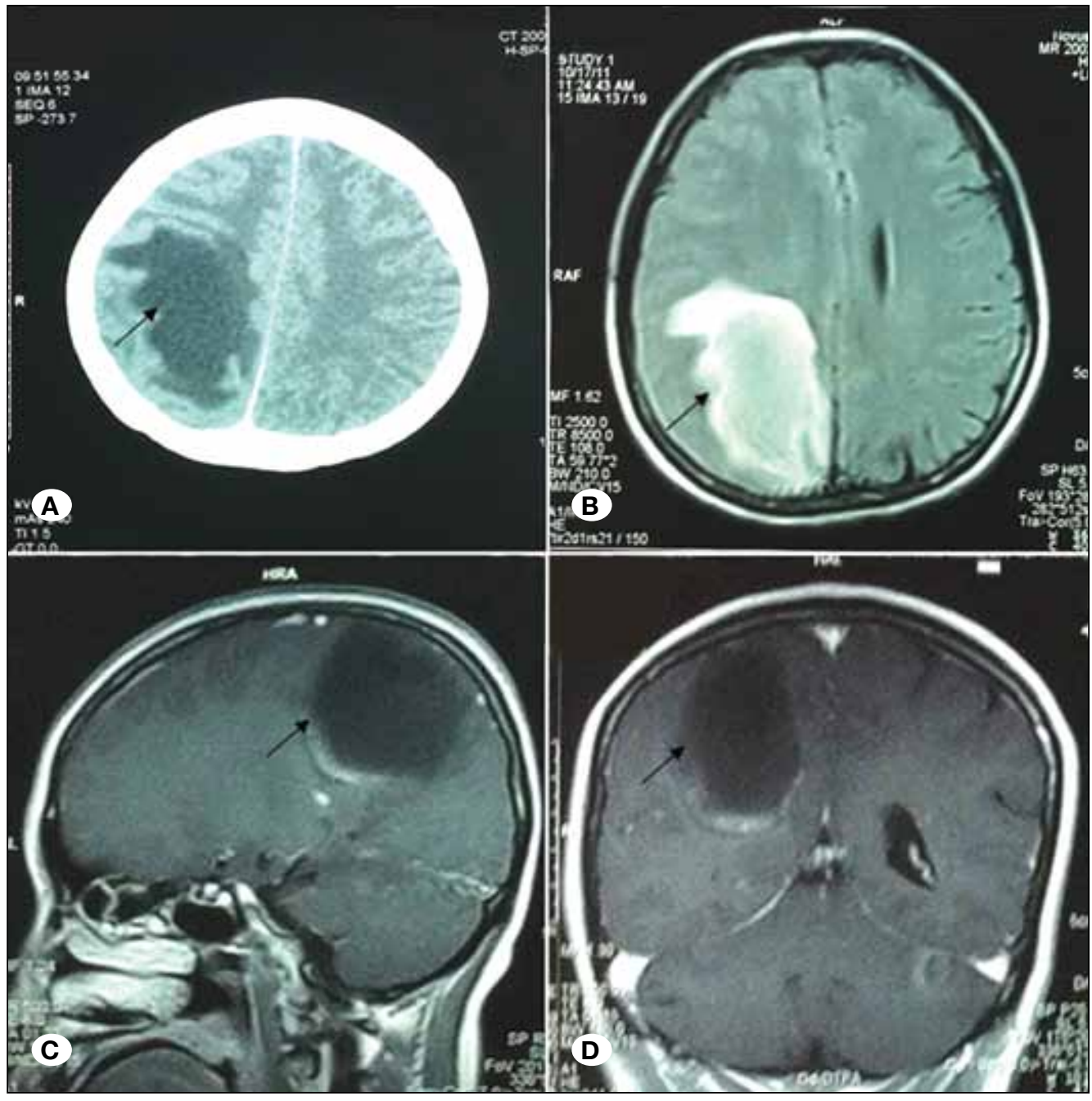

Figure 1: CT and MRI before Treatment.

A) Axial CT showed a large low density area at the right parietal lobe (arrow).

B) MRI with FLAIR showed an abnormal signal at the right parietal lobe (arrow).

C, D) Enhanced scans showed that the edge of the "lump" was in the sagittal plane and a cordlike enhancement (arrow) was distributed vertical to the lateral ventricles in the coronal plane. 

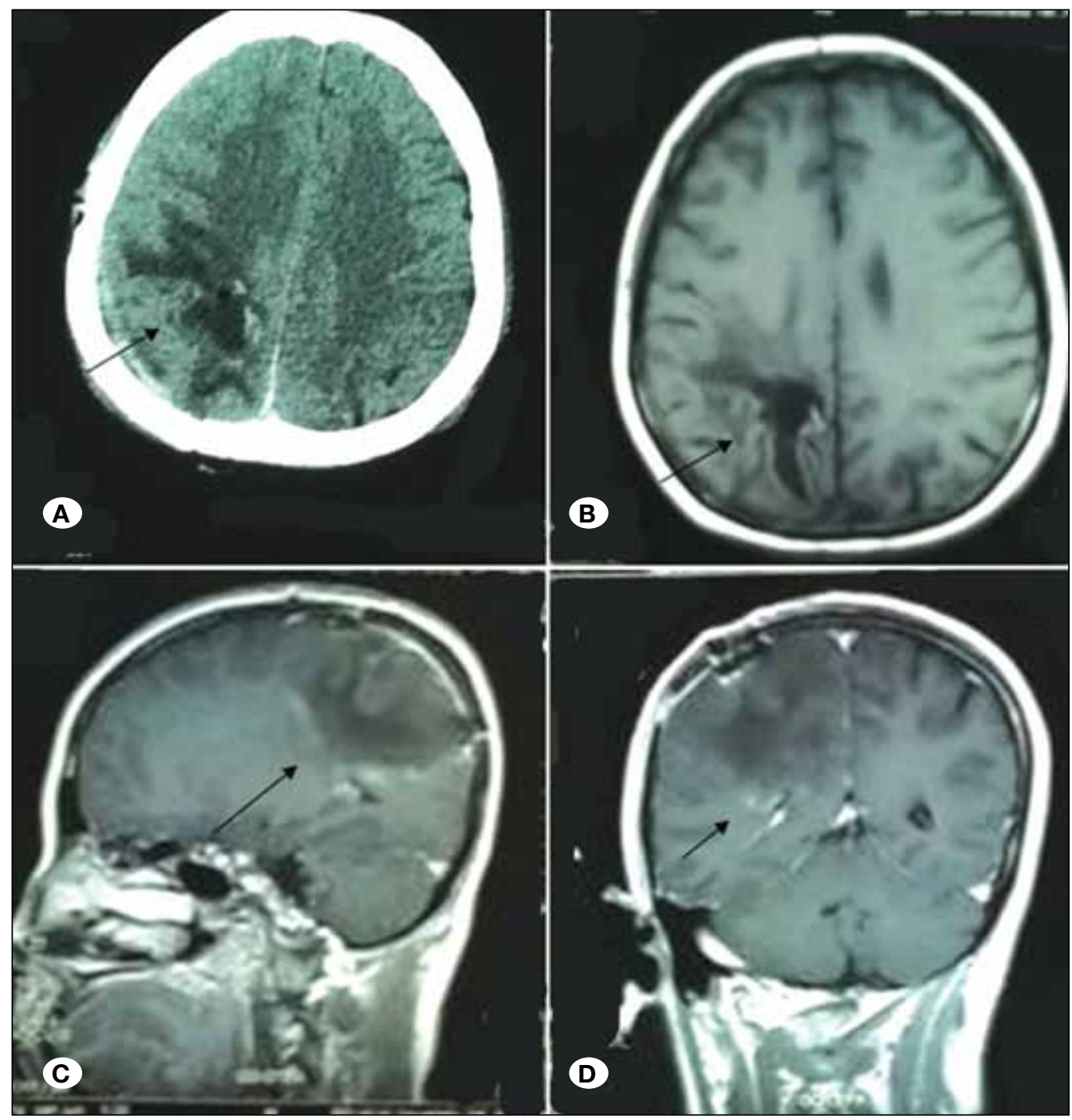

Figure 2: CT and MRI after Treatment.

A, B) Axial CT and MR images showed that the large low density area at the right parietal lobe disappeared (arrow). C, D) Enhanced MR image showed that the "lump" and the cord-like enhancement of the edge of the "lump" disappeared (arrow).

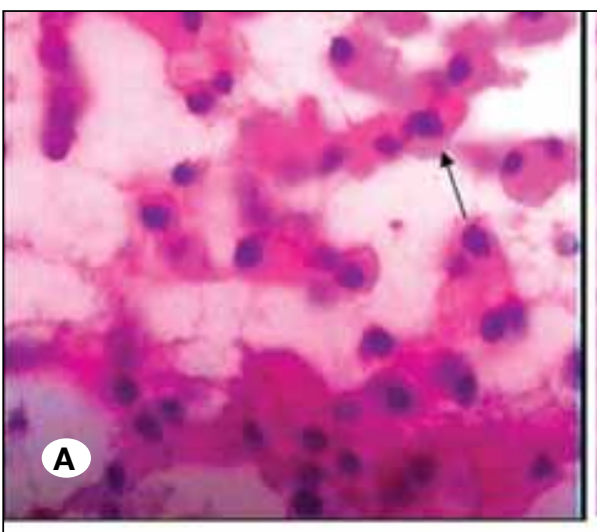

HE x100

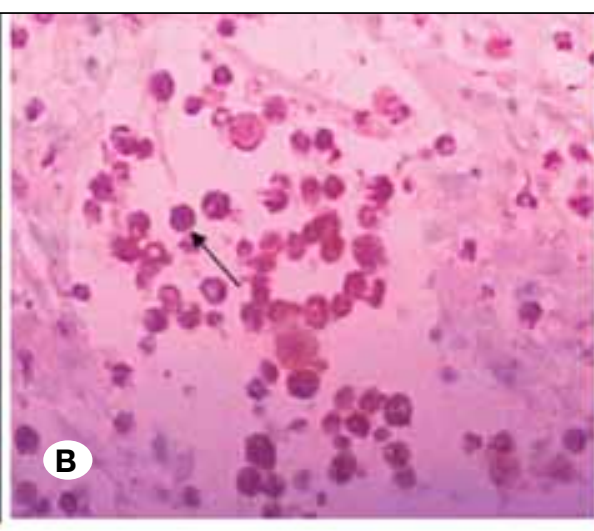

CD68 x100

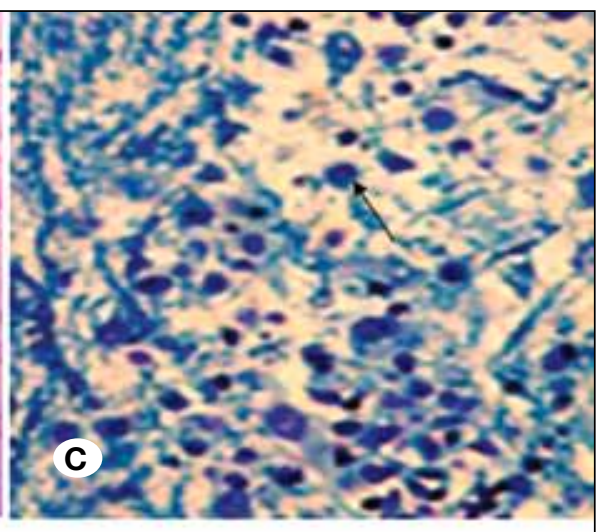

Luxol x100

Figure 3: Pathologic finding revealed demyelinating pseudotumor. A) HE staining of the removed brain tissue, which showed accumulations of macrophages (arrow). B) Macrophages (arrow), but no swollen astrocytes were detected with CD86 staining. C) Luxol fast stains showed some fiber and extensive destruction of myelin with macrophage infiltration (arrow). 
shown that demyelinating pseudotumor can occur at any age with an increased tendency in young and middle-aged females $(8,20)$. The patient in this report was a 40 -year-old female. There are no specific radiology and clinical manifestations of intracranial demyelinating pseudotumor and the accurate diagnosis relies on pathology results. In 1979, van der Velden et al. (17) first described this disease. Pathological features included different degrees of demyelination in the lesion area, axonal reservation, astrocytosis, and perivascular infiltration with a large number of mononuclear macrophages and lymphocytes. Most of the patients showed a single lesion with or without old lesions at the same site. Demyelinating pseudotumor progresses with an acute and severe onset phase that can later gradually stabilize with prolonged disease duration (7). Quick frozen sections are often misdiagnosed as astrocytoma because it is difficult to distinguish macrophages and swollen astrocytes. Therefore, Xia et al. (19) suggested that intraoperative frozen section analysis was inconclusive in the diagnosis of intracranial demyelinating pseudotumor. Immunohistochemical techniques should be applied when the case is suspicious or uncertain (3). Immunohistochemical examinations of myelin and CD68 staining can be used to confirm the diagnosis of intracranial demyelinating pseudotumor because the CD68 marker discriminates between macrophages and swollen astrocytes (3). The observation of demyelination and axonal preservation, combined with CD68 staining indicates intracranial demyelinating pseudotumor. In the current patient, CD68 staining was positive, there was extensive destruction of myelin with macrophage infiltration, and preserved axonal fibers. Therefore, the patient was diagnosed with intracranial demyelinating pseudotumor and treated with steroids. Based on the last follow-up, the patient had recovered well.

Intracranial demyelinating pseudotumor often presents as a single-subcortical lesion with an unclear boundary mass without obvious surrounding edema and significant disproportion between the mass effect and lesion volume. In most cases, CT imaging shows low or modest densities, while MRI shows abnormal long T1 and T2 signals. In the literature, the majority of demyelinating pseudotumor cases presented with non-closed ring enhancements (also called crescent sign) on CT and (or) MRI scans $(11,12)$. However, intracranial tumors often lack this signal $(13,18)$. Ma et al. (10) suggested that non-closed ring enhancements observed in demyelinating pseudotumor tend to be perpendicular to the surface of the lateral ventricle. In the differential diagnosis of astrocytoma, single demyelinating pseudotumor should always be ruled out.

Low grade astrocytoma usually presents on CT and MRI scans without any enhancement or is only slightly enhanced, however, demyelinating pseudotumor presents with significant enhancement. In addition, complete ring or lace-like enhancements with uneven thickness are found in the acute phase of high grade astrocytoma. Unlike astrocytoma, demyelinating pseudotumor is often associated with light edema and less mass effect.
Demyelinating pseudotumor also should be differentiated from other demyelinating lesion such as multiple sclerosis. Multiple sclerosis often shows multiple and symmetrically located lesions in a periventricular distribution (2). It should be noted, that the patient in our case presented with a cord-like enhancement and non-typical MRI features for demyelinating pseudotumor.

In the clinic, demyelinating pseudotumors are often sensitive to hormone therapy with decreased recurrence after treatment. Kepes described 31 cases of demyelinating pseudotumor treated with hormone therapy, where 28 out of 31 cases showed no recurrence at follow-up 9 months to 12-year posttreatment (8). This finding suggests that the prognosis in the majority of demyelinating pseudotumor patients is very good. Thus, if we can timely and correctly diagnose demyelinating pseudotumor, patients will receive treatment earlier and avoid blind surgery, radiotherapy and chemotherapy that could cause unnecessary physical damage and economic losses.

Although the case was misdiagnosed, we were able to outline how to differentially diagnose demyelinating pseudotumor and astrocytoma based on our case and the literature $(1,9,15,16,21)$.

\section{- CONCLUSION}

1) Cerebral demyelinating pseudotumor is often found with an acute onset and short duration, while astrocytomas show a slower progression. Consequently, the presented case had a medical history of only one week;

2) Patients with demyelinating pseudotumor are often young and middle-aged. Our patient was a 40-year old patient;

3) Unlike astrocytoma, demyelinating pseudotumor is often accompanied with light edema and less mass effect, which can be observed on CT and MRI scans;

4) Lesion enhancements of demyelinating pseudotumor tend to be perpendicular to the surface of the lateral ventricle;

5) Demyelinating pseudotumors are often sensitive to hormone therapy with less recurrence risk after treatment. All the above mentioned features should be kept in mind and should be used when a demyelinating pseudotumor cannot be excluded from the differential diagnosis.

\section{- REFERENCES}

1. Akimoto J, Nakajima N, Saida A, Haraoka J, Kudo M: Monofocal acute inflammatory demyelination manifesting as open ring sign. Case report. Neurol Med Chir (Tokyo) 46 (7):353-357,2006

2. Basic Kes V, Cesarik M, Coric L, Zavoreo I, Rotim K, Beros V, Pazanin, L. Drnasin S, Demarin V: Tumor-like multiple sclerosis. Acta Clin Croat 51(1):113-116,2012

3. Erana-Rojas IE, Barboza-Quintana A, Ayala AG, Fuller GN: Demyelinating pseudotumor. Ann Diagn Pathol 6(5):265271,2002

4. Francis G: Large focal tumor-like demyelinating lesions of the brain. Ann Neurol 34(6):871-873,1993 
5. Gutrecht JA, Berger JR, Jones RH Jr, Mancall AC: Monofocal acute inflammatory Demyelination (MAID): A unique disorder simulating brain neoplasm. South Med J 95(10):11801186,2002

6. Huisman TA: Tumor-like lesions of the brain. Cancer Imaging 9 Spec No A:S10-13,2009

7. Jaffe SL, Minagar A: Demyelinating pseudotumor. Arch Neurol 62(9):1466-1467,2005

8. Kepes JJ: Large focal tumor-like demyelinating lesions of the brain: intermediate entity between multiple sclerosis and acute disseminated encephalomyelitis? A study of 31 patients. Ann Neurol 33(1):18-27,1993

9. Kimura N, Kumamoto T, Hanaoka T, Hasama Y, Nakamura $\mathrm{K}$, Okazaki T: Monofocal large inflammatory demyelinating lesion, mimicking brain glioma. Clin Neurol Neurosurg 111(3): 296-299,2009

10. Ma L, Cai YQ, Gao YG: The central nervous system of demyelinating pseudotumor MRI performance. Chinese Journal of Radiology 36(7):601-604,2002

11. Masdeu JC, Moreira J, Trasi S, Visintainer P, Cavaliere $\mathrm{R}$, Grundman $\mathrm{M}$ : The open ring. A new imaging sign in demyelinating disease. J Neuroimaging 6(2):104-107,1996

12. Masdeu JC, Quinto C, Olivera C, Tenner M, Leslie D, Visintainer $P$ : Open-ring imaging sign: Highly specific for atypical brain demyelination. Neurology 54(7):1427-1433,2000

13. Okamoto K, Furusawa T, Ishikawa K, Quadery FA, Sasai K, Tokiguchi S: Mimics of brain tumor on neuroimaging: Part I. Radiat Med 22(2):63-76,2004
14. Peterson K, Rosenblum MK, Powers JM, Alvord E, Walker RW, Posner JB: Effect of brain irradiation on demyelinating lesions. Neurology 43(10):2105-2112,1993

15. Salunke P, Aggarwal A, Gupta K, Agrawal P, Ahuja CK, Vasishta RK: Large demyelinating lesions: A neurosurgical perspective. Br J Neurosurg 26(4):490-498,2012

16. Uematsu Y, Fukai J, Okita R, Owai Y, Fujita K, Tanaka Y, Itakura T: Intra-axial pseudotumors in the central nervous system: Clinicopathological analysis. Brain Tumor Pathol 27 (2):71-80,2010

17. van der Velden M, Bots GT, Endtz LJ: Cranial CT in multiple sclerosis showing a mass effect. Surg Neurol 12(4):307310,1979

18. Wolf J, Shenep JL, Clifford V, Curtis N, Flynn PM: Ethanol lock therapy in pediatric hematology and oncology. Pediatr Blood Cancer 60(1):18-25,2013

19. Xia L, Lin S, Wang ZC, Li SW, Xu L, Wu J, Hao SY, Gao CC: Tumefactive demyelinating lesions: Nine cases and a review of the literature. Neurosurg Rev 32(2):171-179; discussion 179,2009

20. Zagzag D, Miller DC, Kleinman GM, Abati A, Donnenfeld $H$, Budzilovich $G N$ : Demyelinating disease versus tumor in surgical neuropathology. Clues to a correct pathological diagnosis. Am J Surg Pathol 17(6):537-545,1993

21. Ziad F, Katchy KC, Panda SM, Alexander S, Al-Sheikh T: Inflammatory demyelinating pseudotumor. Neurosciences (Riyadh) 11(3):191-193,2006 